\title{
35. AMINO ACIDS IN INTERSTITIAL WATERS FROM SITES 790 AND 791 IN THE IZU-BONIN ISLAND ARC ${ }^{1}$
}

\author{
Hodaka Kawahata ${ }^{2}$ and Toshio Ishizuka ${ }^{3}$
}

\begin{abstract}
Sites 790 and 791 lie in the eastern half graben of the Sumisu Rift, a backarc graben west of the active Izu-Bonin arc volcanoes Sumisu Jima and Tori Shima, at $30^{\circ} 54.96{ }^{\circ} \mathrm{N}, 139^{\circ} 50.66^{\circ} \mathrm{E}$, in $2223 \mathrm{~m}$ water depth and $30^{\circ} 54.97^{\circ} \mathrm{N}, 139^{\circ} 52.20^{\circ} \mathrm{E}$, in $2268 \mathrm{~m}$ water depth, respectively. A small decrease in the sulfate concentration in the interstitial waters from these sites suggests fairly low microbial activity by sulfate-reducing bacteria.

The values of the dissolved free amino acids (DFAA) in the interstitial waters from both sites range from 1.26 to $6.82 \mu \mathrm{mol} / \mathrm{L}$, with an average of $3.81 \mu \mathrm{mol} / \mathrm{L}$. The acidic, basic, neutral, aromatic, and sulfur-containing amino acids have average values of $0.32,0.50,2.71,0.15$, and $0.09 \mu \mathrm{mol} / \mathrm{L}$, respectively. The relative abundances of the acidic, basic, neutral, aromatic, and sulfur-containing amino acids average $8,13,72,4$, and $1 \mathrm{~mol} \%$, respectively. Glycine, serine, alanine, ornithine, and aspartic acid are major constituent amino acids. The dissolved combined amino acids (DCAA) values range between 1.25 and $44.35 \mu \mathrm{mol} / \mathrm{L}$, with an average of $10.36 \mu \mathrm{mol} / \mathrm{L}$. The mean concentrations and relative abundances of the acidic, basic, neutral, aromatic, and sulfur-containing amino acids are $2.29(22 \mathrm{~mol} \%), 0.60(6 \mathrm{~mol} \%), 6.70(65 \mathrm{~mol} \%), 0.09(1 \mathrm{~mol} \%)$, and $0.00 \mu \mathrm{mol} / \mathrm{L}(0 \mathrm{~mol} \%)$, respectively. Glycine is the most abundant amino acid residue, followed by glutamic acid, serine, and alanine. The predominance of DCAA over DFAA present in the interstitial waters from Sites 790 and 791 is consistent with previous results from interstitial-water and seawater analyses.

The most plausible source for the DCAA is biogenic calcareous debris. A much greater depletion of aspartic acid and the basic fraction, except for ornithine, is found in the DCAA. The decomposition of the basic amino acid fraction or its incorporation to clay minerals would result in a decrease in its relative abundance, whereas ornithine is produced during early diagenesis. The characteristics of the amino acids in the interstitial waters are (1) a greater depletion of the acidic amino acid fraction in the DFAA than in the DCAA and (2) the enrichment of glycine and serine in both. The adsorption or reaction of the amino acids in interstitial waters with biogenic carbonates would be responsible for the lower relative abundance of the acidic fraction of the DFAA. The production of glycine during early diagenesis and its stability in solution would raise its relative abundance in the interstitial waters.
\end{abstract}

\section{INTRODUCTION}

Amino acids are common to all organisms and major nitrogenous compounds in sediments and interstitial waters (Degens, 1970; Henrichs and Farrington, 1979). Total sediment hydrolyzable amino acids usually decompose more rapidly than total sediment organic carbon (e.g., Whelan, 1977; Malta et al., 1982). However, the composition of the total sediment hydrolyzable amino acids rarely changes significantly with depth (Rosenfeld, 1979; Henrichs et al., 1984). In contrast, both the concentration and composition of the dissolved free amino acids (DFAA) change markedly with depth (Henrichs and Farrington, 1979; Jørgensen et al., 1981; Henrichs et al., 1984). This difference reflects the differing time scale of supply and removal: tens of years or more for total sediment organic carbon and total sediment hydrolyzable amino acid, but less than one day for DFAA (Christensen and Blackburn, 1980).

Because the amino acid concentrations in interstitial waters are much lower than those in sediments, even small changes of the latter would lead to large changes of the former. Thus, the dissolved combined amino acids (DCAA) in interstitial waters as well as the DFAA seem to be sensitive and important indicators of early diagenesis. However, little work on this subject, particularly on DCAA in interstitial waters, has been done (Ishizuka et al., 1988; Kawahata and Ishizuka, 1989; Kawahata et al., 1990; Ishizuka et al., 1990). Therefore, this paper presents the results of the DFAA, DCAA, and total hydrolyzable amino acids (THAA) in interstitial waters collected

\footnotetext{
'Taylor, B., Fujioka, K., et al., 1992. Proc. ODP, Sci. Results, 126: College Station, TX (Ocean Drilling Program).

${ }^{2}$ Geological Survey of Japan, 1-1-3 Higashi, Tsukuba, Ibaraki 305, Japan.

${ }^{3}$ Ocean Research Institute, University of Tokyo, 1-15-1 Minamidai, Nakano, Tokyo 164, Japan.
}

during Ocean Drilling Program (ODP) Leg 126 and discusses their characteristics and the relationship among them.

\section{METHODS}

\section{Sampling and Storage}

Interstitial waters from the sediments at Sites 790 and 791 were collected aboard JOIDES Resolution by hydraulic squeezing. All interstitial-water samples were filtered through $0.22-\mu \mathrm{m}$ Millepore cellulose acetate filters in the shipboard laboratory. For amino acid analysis of the interstitial water, a 5-mL aliquot of the water sample was placed in a precombusted glass ampule. After being flushed with helium, the ampule was sealed and stored refrigerated $\left(1^{\circ}-2^{\circ} \mathrm{C}\right)$ until delivery to the land-based laboratory.

\section{Analytical Procedures: Amino Acids}

The DFAA in the interstitial waters were analyzed after the addition of $7 \mu \mathrm{L}$ of ultrapure (amino-acid-free) $17 \% \mathrm{HCl}$ to $0.7 \mathrm{~mL}$ of an interstitial-water sample. Amino acid concentrations were determined by direct injection of the sample into an automated liquid chromatograph (Hitachi model 835). The reported concentrations have been corrected for the reagent blank.

For total hydrolyzable amino acids (THAA), $1 \mathrm{~mL}$ of interstitial water, together with $1 \mathrm{~mL}$ of ultrapure concentrated $\mathrm{HCl}$ to adjust the solution to $6 \mathrm{~N} \mathrm{HCl}$, was placed in a precombusted glass ampule and hydrolyzed at $110^{\circ} \mathrm{C}$ for $22 \mathrm{hr}$ under an argon atmosphere. Then, the solution was evaporated gently using a rotary evaporator at a temperature lower than $42^{\circ} \mathrm{C}$ and the residue was redissolved in $1 \mathrm{~mL}$ of $0.01 \mathrm{~mol} \mathrm{HCl}$. This aliquot was injected into the amino acid analyzer. The standard deviation for amino acid analysis based on replicated measurements of a standard solution (about $1 \mu \mathrm{mol} / \mathrm{L}$ for amino acids) 
was $<10 \%$, and the detection limit of our method was about $0.05 \mu \mathrm{mol} / \mathrm{L}$. Low values of DFAA and THAA near the detection limit may not be dependable. Nevertheless, we retain these values because they confirm that the amino acid concentration is low (Ishizuka et al., 1988).

\section{SUMMARY OF THE GEOLOGICAL FRAMEWORK}

Sites 790 and 791 lie in the eastern half graben of the Sumisu Rift, a backarc graben west of the active Izu-Bonin arc volcanoes Sumisu Jima and Tori Shima (Fig. 1). Site 791 was drilled on the western edge of the flat basin floor of the graben, whereas Site 790 is situated on a slight rise and structural high to the west of the flat basin floor. The sites are $2.4 \mathrm{~km}$ apart.

Site 790 is at $30^{\circ} 54.96^{\prime} \mathrm{N}, 139^{\circ} 50.66^{\prime} \mathrm{E}$, at $2223 \mathrm{~m}$ below sea level (mbsl). The stratigraphic succession consists of three lithologic units composed predominantly of volcanogenic materials; the upper two units are sedimentary, whereas the basal unit is igneous. The sedimentary units also contain variable quantities of biogenic materials, mainly nannofossils (Table 1). Site 791 is at $30^{\circ} 54.97 \mathrm{~N}, 139^{\circ} 52.20^{\prime} \mathrm{E}$, at $2268 \mathrm{mbsl}$. The succession is divided into three lithologic units; the upper two units are sedimentary and the lower one is igneous (Table 1).

Sedimentation rates determined from calcareous nannofossil and radiolarian datums and from paleomagnetic events at each site exhibit an exponential increase over the recovered interval. At Site 791, sedimentation rates increase from $344 \mathrm{~m} / \mathrm{m}$.y. at the base of Unit II (approximately $1 \mathrm{Ma}$ ) to more than $2200 \mathrm{~m} / \mathrm{m}$.y. at the top of Unit I. At Site 790 , sedimentation rates increase from $90 \mathrm{~m} / \mathrm{m}$.y. at about $1 \mathrm{Ma}$ to about $1000 \mathrm{~m} / \mathrm{m}$.y. at present. Before $0.275 \mathrm{Ma}$, sedimentation rates at Site 791 are approximately 4 times those found at Site 790. Between 0.275 and $0.10 \mathrm{Ma}$, linear sedimentation rates at Site 791 are greater by a factor of 3 (Table 1) (Shipboard Scientific Party, 1990).

Average total organic carbon (TOC) and inorganic carbon (carbonate) are 0.40 and $1.85 \mathrm{wt} \%(15.4 \mathrm{wt} \%)$ at Site 790 and 0.45 and $2.24 \mathrm{wt} \%$ (18.6 wt\%) at Site 791 (Shipboard Scientific Party, 1990).

\section{INORGANIC CHEMISTRY OF THE INTERSTITIAL WATERS}

The chlorinity and $\mathrm{pH}$ are about $514 \mathrm{mmol} / \mathrm{L}$ and 7.57 , respectively, and are fairly constant throughout the holes drilled at Site 790. The concentrations of sulfate decrease gradually from 27.9 to 15.1 $\mathrm{mmol} / \mathrm{L}$ in the upper $200 \mathrm{~m}$ and increase to $22.9 \mathrm{mmol} / \mathrm{L}$ at the bottom, which suggests low activity of sulfate-reducing bacteria. The profile of calcium is similar to that of sulfate. Average concentrations of calcium, magnesium, ammonium, and silica are $9.2 \mu \mathrm{mol} / \mathrm{L}, 52.4$ $\mathrm{mmol} / \mathrm{L}, 754 \mu \mathrm{mol} / \mathrm{L}$, and $699 \mu \mathrm{mol} / \mathrm{L}$, respectively.

Chlorinity, $\mathrm{pH}$, and sulfate concentration are fairly constant throughout the holes of Site 791. Their mean values are $565 \mathrm{mmol} / \mathrm{L}$, 7.60 , and $27.2 \mathrm{mmol} / \mathrm{L}$, respectively. The calcium concentration is at about $20 \mathrm{mmol} / \mathrm{L}$ in the upper $40 \mathrm{~m}$, and it decreases abruptly to about $10 \mathrm{mmol} / \mathrm{L}$ between 40 and $200 \mathrm{~m}$ below seafloor (mbsf) and gradually increases below this depth. Magnesium concentration vs. subbottom depth shows a reversed profile of the calcium concentration. The $\mathrm{Mg}$ concentration is about $45 \mathrm{mmol} / \mathrm{L}$ in the upper $40 \mathrm{~m}$, increases abruptly to about $53 \mathrm{mmol} / \mathrm{L}$ between 40 and $200 \mathrm{mbsf}$, and decreases gradually below this depth. The average concentrations of ammonium and silica are 363 and $543 \mu \mathrm{mol} / \mathrm{L}$, respectively (Shipboard Scientific Party, 1990).

\section{RESULTS}

\section{Site 790}

The concentrations of THAA in the interstitial waters are given in Table 2 and are plotted vs. sub-bottom depth in Figure 2. The THAA values range between 3.53 and $19.36 \mu \mathrm{mol} / \mathrm{L}$ and average 8.18 $\mu \mathrm{mol} / \mathrm{L}$. Neutral amino acids are the most abundant fraction of the

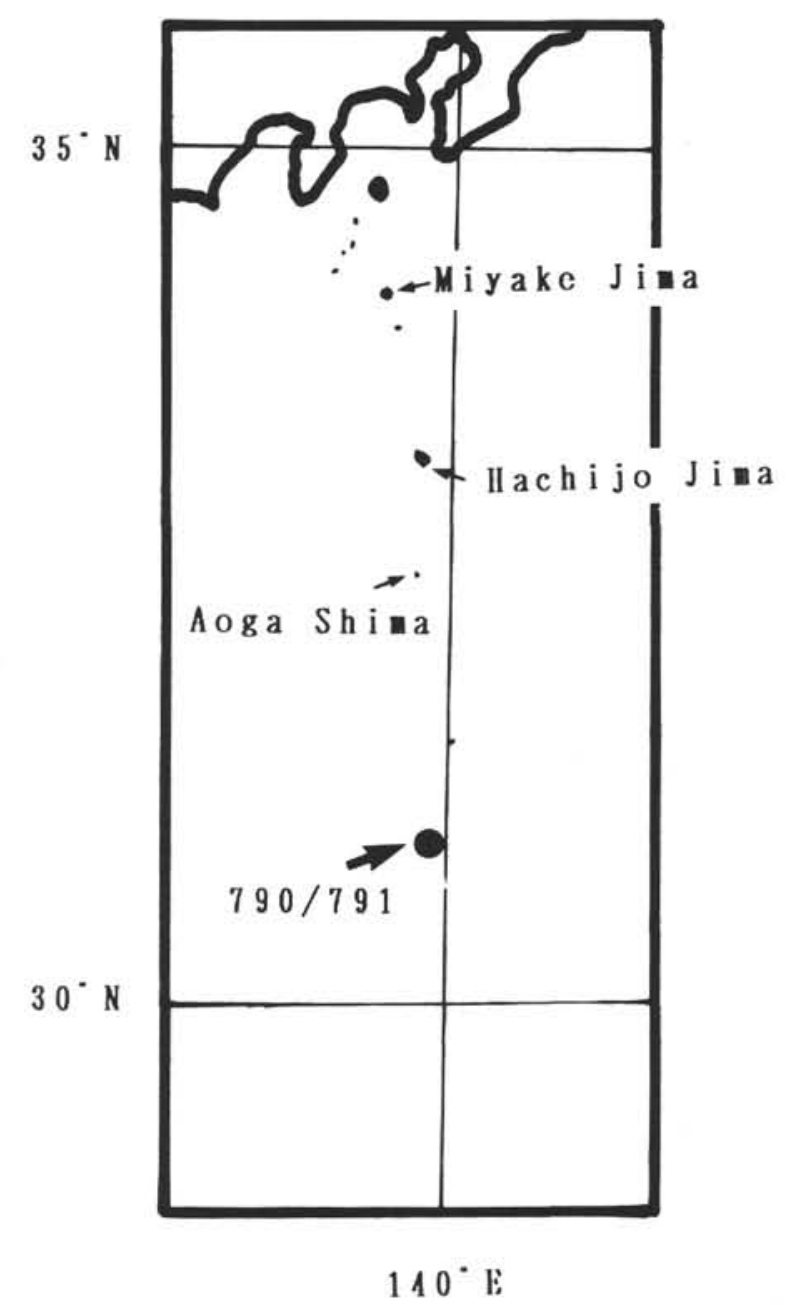

Figure 1. Location of Sites 790 and 791.

amino acids and account for $62 \%$ of the total THAA. The average concentration is $5.10 \mu \mathrm{mol} / \mathrm{L}$. The second most abundant fraction is the acidic amino acids. The mean concentration is $1.68 \mu \mathrm{mol} / \mathrm{L}$, which constitutes $21 \mathrm{~mol} \%$ of the total THAA. The basic amino acid fraction is $0.72 \mu \mathrm{mol} / \mathrm{L}$ on average, which accounts for $9 \%$ of the total THAA. The aromatic and sulfur-containing amino acids are minor fractions. Their mean values are 0.11 and $0.09 \mu \mathrm{mol} / \mathrm{L}$, respectively. The relative abundances of aromatic and sulfur-containing amino acids are each only $1 \mathrm{~mol} \%$ of the total THAA. The THAA composition shows no consistent trend with sub-bottom depth or with the total concentration. Glycine is the most abundant amino acid residue, with glutamic acid, serine, and ornithine next. The $\gamma$ and $\alpha$-amino butyric acids and $\beta$-alanine of nonprotein amino acids constitute about 2,1 , and $1 \mathrm{~mol} \%$ of the total THAA, respectively.

The DFAA concentrations in the interstitial waters are given in Table 3 and Figure 3 and are plotted vs. sub-bottom depth in Figure 2. Total DFAA values range from 1.26 to $6.82 \mu \mathrm{mol} / \mathrm{L}$ and average $3.41 \mu \mathrm{mol} / \mathrm{L}$. Average values of the acidic, basic, neutral, aromatic, and sulfur-containing amino acids are $0.32,0.40,2.50,0.07$, and 0.09 $\mu \mathrm{mol} / \mathrm{L}$, respectively. The relative abundances of acidic, basic, neutral, and aromatic amino acids average $9,12,73,12$, and $2 \mathrm{~mol} \%$, respectively. Glycine, serine, alanine, and ornithine are major amino acids of DFAA. The DFAA shows that the profile is similar to that of THAA. Relatively high concentration values are found at the near surface (131 and $255 \mathrm{mbsf}$ ), which do not always correspond to high values of TOC and $\mathrm{CaCO}_{3}$ in the sediments. 
Table 1. Characteristics of lithologic units, Sites 790 and 791.

\begin{tabular}{|c|c|c|c|c|c|c|}
\hline Unit & $\begin{array}{l}\text { Depth } \\
\text { (mbsf) }\end{array}$ & Lithology & Age & $\begin{array}{c}\text { Porosity } \\
(\%)\end{array}$ & $\begin{array}{l}\text { Density } \\
\left(\mathrm{g} / \mathrm{cm}^{3}\right)\end{array}$ & $\begin{array}{c}\text { Sedimentation } \\
\text { rate } \\
(\mathrm{m} / \mathrm{m} . \mathrm{y} .)\end{array}$ \\
\hline \multicolumn{7}{|l|}{ Site 790} \\
\hline 1 & $0-165$ & $\begin{array}{l}\text { Vitric silt, vitric sand, pum- } \\
\text { iceous gravel, clayey silt, } \\
\text { and silty clay. Gravels are } \\
\text { felsic; sands are mafic and } \\
\text { felsic. Triplet beds of } \\
\text { black sand/silt, light gray } \\
\text { vitric clayey silt, and bur- } \\
\text { rowed clayey silt with } \\
\text { pumice and basaltic } \\
\text { grains. }\end{array}$ & Quatemary & 67 & 2.6 & 717 \\
\hline II & $165-266.6$ & $\begin{array}{l}\text { Nannofossil clay, nannofossil- } \\
\text { rich clay, silty clay, and } \\
\text { clayey silt. Thin ash beds. } \\
\text { Scattered pumice and sco- } \\
\text { ria clasts. Triplet beds } \\
\text { from } 165 \text { to } 184 \text { mbsf as } \\
\text { in Unit I. }\end{array}$ & Quaternary & 66 & 2.7 & 131 \\
\hline III & $266.6-387$ & Scoriaceous basalt. & Quatemary & - & - & - \\
\hline \multicolumn{7}{|l|}{ Site 791} \\
\hline I & $0-428.4$ & $\begin{array}{l}\text { Pumiceous gravel, pumiceous } \\
\text { pebbly sand and sand, } \\
\text { vitric silt with minor clay, } \\
\text { silty clay, and nannofossi- } \\
\text { rich silty clay. }\end{array}$ & Quatemary & 66 & 2.5 & 1930 \\
\hline II & $428.4-834$ & $\begin{array}{l}\text { Nannofossil-rich claystone } \\
\text { and nannofossil claystone } \\
\text { with scattered volcanic } \\
\text { grains, particularly below } \\
680 \text { mbsf; nannofossil } \\
\text { sandy mudstone; and } \\
\text { vitric silt. Pyrite occurs in } \\
\text { burrows. }\end{array}$ & Quatemary & 59 & 2.7 & 522 \\
\hline III & $834-1145$ & $\begin{array}{l}\text { Basalt, diabase, basaltic } \\
\text { mousse, felsic ash to } \\
\text { lapilli tuff, and rare dark } \\
\text { vitric silt. }\end{array}$ & Quaternary & - & - & - \\
\hline
\end{tabular}

Note: Mean values from Shipboard Scientific Party (1990).

Hydrolyzable

\section{Relative abundance Total $(\mu \mathrm{M} / \mathrm{L})$ Relative abundance Total $(\mu \mathrm{M} / \mathrm{L})$}
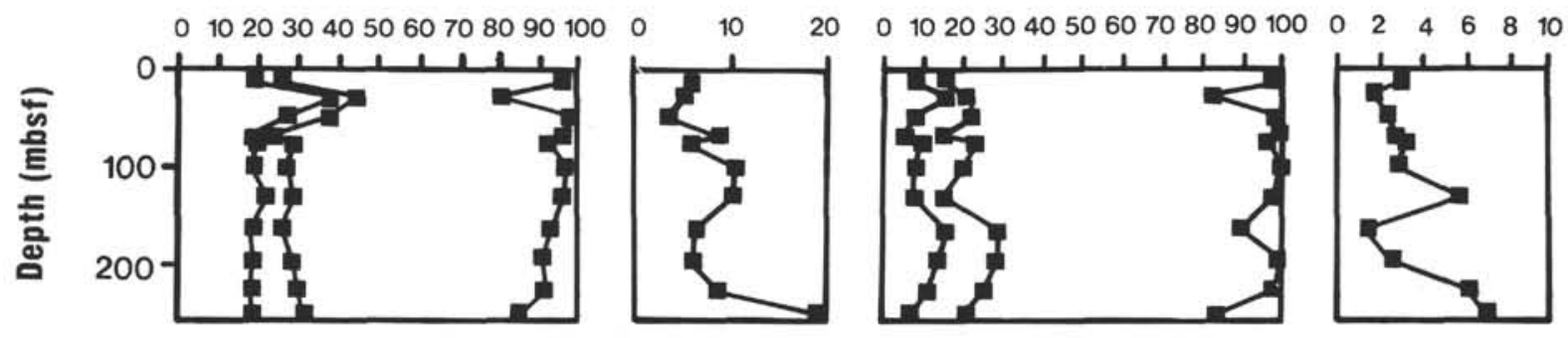

Figure 2. Concentrations of acidic, basic, neutral, and total amino acids of THAA and DFAA in interstitial waters vs. depth, Site 790.

\section{Site 791}

Concentrations of individual THAA are given in Table 2. Total concentrations of THAA range from 6.02 to $49.94 \mu \mathrm{mol} / \mathrm{L}$ and average $20.16 \mu \mathrm{mol} / \mathrm{L}$. In a plot of THAA concentrations vs. subbottom depth (Fig. 4), total THAA values generally decrease vs. sub-bottom depth. In contrast, the contents of $\mathrm{CaCO}_{3}$ in the sediments generally increase vs. sub-bottom depth. Fairly high values are found at depths of 38 and 137 mbsf. In spite of the high fluctuation of the total THAA values, the relative abundance of the individual amino acids is fairly uniform throughout the holes. The mean values of the acidic, basic, neutral, aromatic, and sulfur-containing amino acids are $3.55,1.49,13.74,0.37$, and $0.09 \mu \mathrm{mol} / \mathrm{L}$, respectively. Their mean relative abundances are $18,7,68,2$, and $0.4 \mathrm{~mol} \%$, respectively.

Concentrations of individual DFAA given in Table 3 are plotted vs. sub-bottom depth in Figure 4. Total DFAA concentrations range from 1.74 to $5.83 \mu \mathrm{mol} / \mathrm{L}$ and average $4.21 \mu \mathrm{mol} / \mathrm{L}$. Fairly high values are found in the upper $150 \mathrm{~m}$ and at $813 \mathrm{mbsf}$. The mean values of acidic, basic, neutral, aromatic, and sulfur-containing amino acids are 
Table 2. Concentrations in $\mu \mathrm{mol} / \mathrm{L}$ of total hydrolyzable amino acids (THAA) in interstitial waters, Sites 790 and 791.

\begin{tabular}{|c|c|c|c|c|c|c|c|c|c|c|c|}
\hline \multicolumn{2}{|c|}{$\begin{array}{l}\text { Hole, core, section } \\
\text { Interval }(\mathrm{cm})\end{array}$} & $\begin{array}{c}790 \mathrm{~B}-2 \mathrm{H}-3 \\
140-150\end{array}$ & $\begin{array}{c}790 \mathrm{~B}-3 \mathrm{H}-5 \\
140-150\end{array}$ & $\begin{array}{c}790 \mathrm{~B}-6 \mathrm{H}-3 \\
140-150\end{array}$ & $\begin{array}{c}790 \mathrm{~B}-8 \mathrm{H}-5 \\
140-150\end{array}$ & $\begin{array}{c}790 \mathrm{~B}-9 \mathrm{H}-2 \\
140-150\end{array}$ & $\begin{array}{c}790 \mathrm{C}-2 \mathrm{H}-5 \\
0-5\end{array}$ & $\begin{array}{c}790 \mathrm{C}-5 \mathrm{H}-5 \\
140-150\end{array}$ & $\begin{array}{c}790 \mathrm{C}-10 \mathrm{H}-1 \\
101-111\end{array}$ & $\begin{array}{c}790 \mathrm{C}-13 \mathrm{H}-3 \\
140-150\end{array}$ & $\begin{array}{c}790 \mathrm{C}-16 \mathrm{H}-3 \\
140-150\end{array}$ \\
\hline \multicolumn{2}{|c|}{ Depth (mbsf) } & 13.25 & 25.75 & 46.95 & 69.05 & 74.25 & 100.45 & 130.95 & 165.96 & 196.85 & 227.25 \\
\hline \multicolumn{12}{|c|}{ Acidic } \\
\hline & Aspartic acid & 0.39 & 0.29 & 0.19 & 0.53 & 0.29 & 0.54 & 0.54 & 0.33 & 0.42 & 0.51 \\
\hline \multirow{4}{*}{ Basic } & Glutamic acid & 0.84 & 1.69 & 0.76 & 1.01 & 0.80 & 1.37 & 1.78 & 0.79 & 0.70 & 1.07 \\
\hline & Omithine & 0.34 & 0.18 & 0.38 & 0.44 & 0.48 & 0.77 & 0.58 & 0.23 & 0.48 & 0.80 \\
\hline & Lysine & - & - & - & 0.03 & 0.03 & 0.08 & 0.03 & 0.14 & 0.04 & 0.05 \\
\hline & Histidine & $\overline{007}$ & 0.08 & - & - & - & $\overline{000}$ & 0.04 & $\overline{0 x}$ & $\bar{n}$ & \\
\hline & Arginine & 0.07 & - & - & - & - & 0.02 & 0.03 & 0.06 & 0.08 & 0.13 \\
\hline \multicolumn{12}{|c|}{$\begin{array}{l}\text { Neutral } \\
\text { Hydroxy }\end{array}$} \\
\hline & Threonine & 0.49 & 0.13 & 0.09 & 0.61 & 0.10 & 0.28 & 0.48 & 0.46 & 0.33 & 0.41 \\
\hline & Serine & 0.78 & 0.10 & 0.59 & 1.23 & 0.73 & 2.01 & 1.74 & 0.19 & 0.53 & 1.11 \\
\hline \multicolumn{12}{|c|}{ Straight } \\
\hline & $\begin{array}{l}\text { Glycine } \\
\text { Alanine }\end{array}$ & $\begin{array}{l}1.56 \\
0.96\end{array}$ & $\begin{array}{l}0.62 \\
0.25\end{array}$ & 0.21 & $\begin{array}{l}2.58 \\
1.22\end{array}$ & $0.3 x$ & 0.87 & $\begin{array}{l}2.98 \\
1.15\end{array}$ & $\begin{array}{l}1.88 \\
1.34\end{array}$ & $\begin{array}{l}1.67 \\
0.82\end{array}$ & $\begin{array}{l}2.36 \\
0.99\end{array}$ \\
\hline \multicolumn{12}{|c|}{ Branch } \\
\hline & Valine & 0.36 & 0.30 & 0.26 & 0.44 & 0.25 & 0.23 & - & 0.51 & - & - \\
\hline & Iso-teucine & 0,27 & 0.19 & 0.18 & $0.2 x$ & 0.19 & 0.23 & 0.29 & 0.21 & 0.19 & 0.24 \\
\hline & Leucine & 0.19 & 0.17 & 0,11 & 0.25 & 0.14 & 0.21 & 0.26 & 0.21 & 0.18 & 0.21 \\
\hline Other & Aspargine & - & - & - & $=$ & 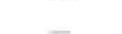 & 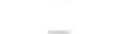 & 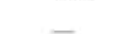 & 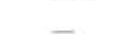 & 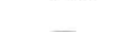 & 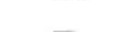 \\
\hline \multicolumn{12}{|c|}{$\begin{array}{l}\text { Aspargine } \\
\text { Aromatic }\end{array}$} \\
\hline & Tyrosine & 0,08 & $0.0 \mathrm{~K}$ & - & 0.15 & 0.11 & 0.05 & 0.21 & 0.05 & 0.06 & 0.18 \\
\hline \multicolumn{12}{|c|}{ Sulfur Phenylalanine } \\
\hline & Cystein & 0.02 & - & - & - & - & - & - & - & - & - \\
\hline \multirow{7}{*}{ Other } & Methionine & - & 一 & - & - & - & - & - & - & - & - \\
\hline & Taurine & - & 0.22 & - & 0.03 & - & 0.11 & 0.02 & 0.03 & 0.02 & 0.03 \\
\hline & Citrulline & - & 0.08 & - & - & - & - & - & - & - & - \\
\hline & $\alpha$-aminoadipic acid & - & - & - & - & - & - & - & - & - & - \\
\hline & $\begin{array}{l}\alpha \text {-aminobutyric acid } \\
\beta \text {-aminobutyric acid }\end{array}$ & $=$ & $\bar{z}$ & E & E & - & - & $=$ & = & - & - \\
\hline & -aminobutyric acid & 0.13 & $0.0 \mathrm{x}$ & - & 0.24 & 0.24 & 0.12 & $0(k)$ & 0.25 & 0.35 & 0.37 \\
\hline & $\hat{\beta}$-alanine & - & 0.54 & 0.07 & & 0.09 & 0.07 & 0.07 & 0.10 & 0.12 & 0.10 \\
\hline Total & & $6.4 x$ & 5.00 & 3.53 & 8.83 & 5.51 & 10.33 & 10.30 & 6.08 & 6.00 & 8.57 \\
\hline
\end{tabular}

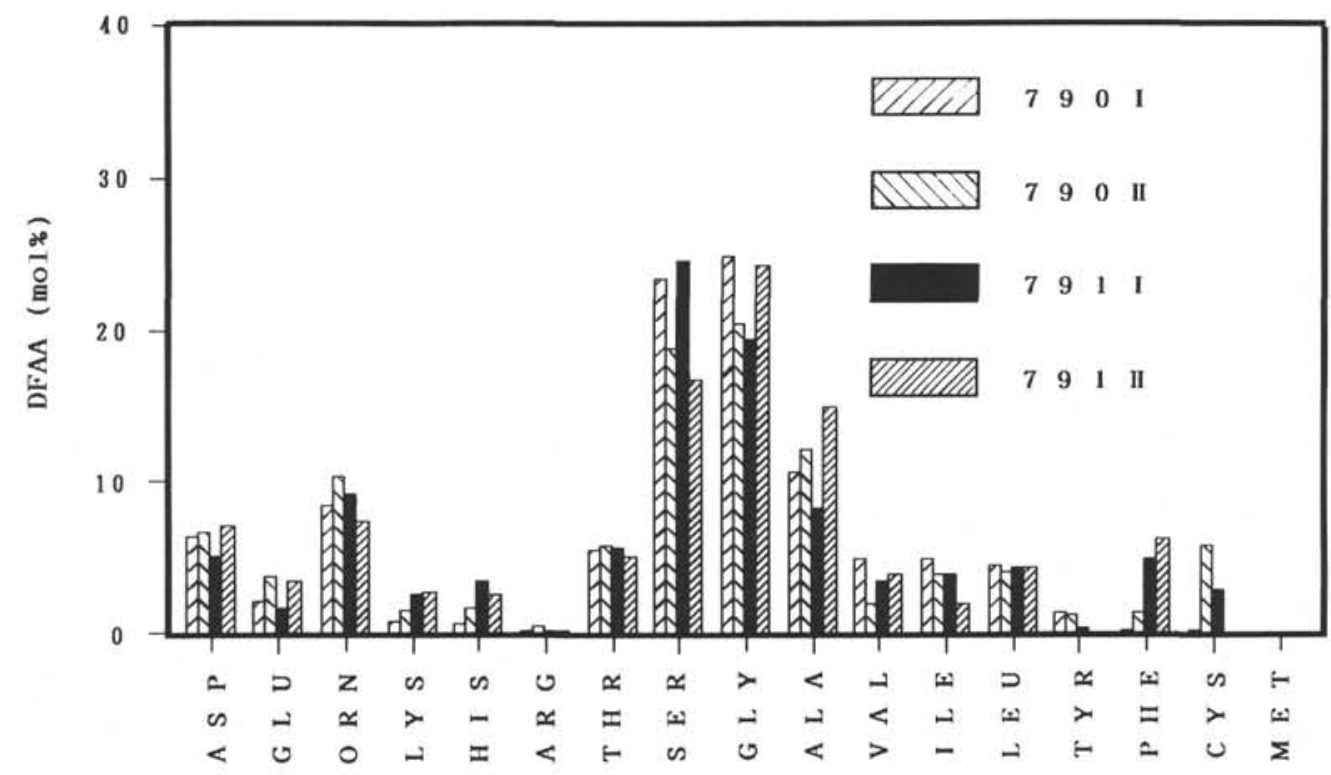

Figure 3. Composition of DFAA in interstitial waters, Sites 790 and 791. The mean mole percentage of each amino acid from all lithologic units at both sites is plotted. Asp = aspartic acid, Glu = glutamic acid, Orn = ornithine, Lys = lysine, His = histidine $\mathrm{Arg}=$ arginine, $\mathrm{Thr}=$ threonine $\mathrm{Ser}=$ serine, $\mathrm{Gly}=$ glycine, $\mathrm{Ala}=$ alanine, $\mathrm{Val}=$ valine, $\mathrm{Ile}=\mathrm{iso}$-leucine, Leu $=$ leucine, $\mathrm{Tyr}=$ tyrosine, $\mathrm{Phe}=$ phenylalanine, $\mathrm{Cys}=$ cysteine, and $\mathrm{Met}=$ methionine .

$0.33,0.61,2.93,0.23,0.08 \mu \mathrm{mol} / \mathrm{L}$, respectively. Their mean relative abundances are $8,14,70,6$, and $2 \mathrm{~mol} \%$, respectively.

\section{DISCUSSION}

\section{DCAA in Interstitial Waters}

Amino acids are present in free and combined states in the interstitial waters. The concentration of DCAA can be calculated by subtracting the DFAA concentration from the THAA concentration in the interstitial waters from Sites 790 and 791 (Table 4 and Fig. 5). The respective concentrations of DCAA and DFAA are, on average, 4.1 and $3.0 \mu \mathrm{mol} / \mathrm{L}$ in lithologic Unit I and 5.9 and $4.1 \mu \mathrm{mol} / \mathrm{L}$ in Unit II at Site 790 and 22.4 and $4.6 \mu \mathrm{mol} / \mathrm{L}$ in Unit $\mathrm{I}$ and 4.6 and $3.5 \mu \mathrm{mol} / \mathrm{L}$ in Unit II at Site 791. The results show a predominance of DCAA over DFAA in the interstitial waters at Sites 790 and 791, which is consistent with previous results from interstitial-water and seawater studies 
Table 2 (continued).

\begin{tabular}{|c|c|c|c|c|c|c|c|c|c|c|c|}
\hline $\begin{array}{c}790 \mathrm{C}-18 \mathrm{H}-2 \\
140-150\end{array}$ & $\begin{array}{c}791 \mathrm{~A}-2 \mathrm{H}-5 \\
140-150\end{array}$ & $\begin{array}{c}791 \mathrm{~A}-5 \mathrm{H}-3 \\
140-150\end{array}$ & $\begin{array}{c}791 \text { A }-9 \mathrm{H}-6 \\
140-150\end{array}$ & $\begin{array}{c}791 \mathrm{~A}-12 \mathrm{H}-5 \\
140-150\end{array}$ & $\begin{array}{c}791 \mathrm{~A}-15 \mathrm{H}-6 \\
25-35\end{array}$ & $\begin{array}{c}791 \mathrm{~A}-18 \mathrm{H}-6 \\
90-100\end{array}$ & $\begin{array}{c}791 \mathrm{~A}-22 \mathrm{H}-6 \\
140-150\end{array}$ & $\begin{array}{c}791 \mathrm{~B}-10 \mathrm{R}-1 \\
86-96\end{array}$ & $\begin{array}{c}791 \mathrm{~B}-13 \mathrm{BR}-1 \\
140-150\end{array}$ & $\begin{array}{c}791 B-23 R-3 \\
115-125\end{array}$ & $\begin{array}{c}791 \mathrm{~B}-45 \mathrm{R}-2 \\
140-150\end{array}$ \\
\hline 254.65 & 11.95 & 37.45 & 800,25 & 107.85 & 1.37.30) & 166.95 & 205.85 & 474.21 & 503.55 & 602.90 & 813.20 \\
\hline $\begin{array}{l}1.16 \\
2.50\end{array}$ & $\begin{array}{l}0.57 \\
1.50\end{array}$ & $\begin{array}{l}2.08 \\
8.13\end{array}$ & $\begin{array}{l}1 .(6) \\
2.84\end{array}$ & $\begin{array}{l}0.84 \\
1.87\end{array}$ & $\begin{array}{l}1.75 \\
5.38\end{array}$ & $\begin{array}{l}0.82 \\
1.93\end{array}$ & $\begin{array}{l}1.04 \\
2.27\end{array}$ & $\begin{array}{l}0.33 \\
1.23\end{array}$ & $\begin{array}{l}0.54 \\
1.37\end{array}$ & $\begin{array}{l}0.40 \\
1.16\end{array}$ & $\begin{array}{l}0.40 \\
1.57\end{array}$ \\
\hline $\begin{array}{l}1.19 \\
0.45 \\
0.49 \\
0.24\end{array}$ & $\begin{array}{l}0.82 \\
0.30 \\
0.24 \\
0.12\end{array}$ & $\begin{array}{l}3.46 \\
0.75 \\
1.01 \\
0.31\end{array}$ & $\begin{array}{l}0.96 \\
0.38 \\
0.19 \\
0.25\end{array}$ & 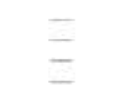 & $\begin{array}{l}2.03 \\
0.47 \\
0.66 \\
0.00\end{array}$ & $\begin{array}{l}0.26 \\
0.24 \\
0.20 \\
0.18\end{array}$ & $\begin{array}{l}0.34 \\
0.37 \\
0.31 \\
0.33\end{array}$ & $\begin{array}{l}0.19 \\
0.12 \\
0.09 \\
0.20\end{array}$ & $\begin{array}{l}0.24 \\
0.17 \\
0.16 \\
-\end{array}$ & $\begin{array}{l}0.03 \\
0.13 \\
=\end{array}$ & $\begin{array}{l}0.57 \\
0.16 \\
0.13 \\
-\end{array}$ \\
\hline $\begin{array}{l}1.07 \\
1.41\end{array}$ & $\begin{array}{l}0.42 \\
3.60\end{array}$ & $\begin{array}{l}1.78 \\
8.67\end{array}$ & $\begin{array}{l}0.61 \\
3.25\end{array}$ & $\begin{array}{l}1.38 \\
6.33\end{array}$ & $\begin{array}{l}1.02 \\
5.00\end{array}$ & $\begin{array}{l}0.35 \\
2.10\end{array}$ & $\begin{array}{l}0.43 \\
2.75\end{array}$ & $\begin{array}{l}0.24 \\
0.73\end{array}$ & $\begin{array}{l}0.42 \\
1.08\end{array}$ & $\begin{array}{l}0.31 \\
0.39\end{array}$ & $\begin{array}{l}0.20 \\
0.74\end{array}$ \\
\hline $\begin{array}{l}4.22 \\
2.07\end{array}$ & $\begin{array}{l}1.93 \\
0.66\end{array}$ & $\begin{array}{r}14.90 \\
3.79\end{array}$ & $\begin{array}{r}10.12 \\
1.51\end{array}$ & $\begin{array}{l}8.17 \\
2.87\end{array}$ & $\begin{array}{r}12.102 \\
2.56\end{array}$ & $\begin{array}{r}10.45 \\
0.81\end{array}$ & $\begin{array}{r}13.48 \\
1.00\end{array}$ & $\begin{array}{l}3.46 \\
0.53\end{array}$ & $\begin{array}{l}3.82 \\
0.68\end{array}$ & $\begin{array}{l}1.72 \\
0.77\end{array}$ & $\begin{array}{l}1.77 \\
0.74\end{array}$ \\
\hline $\begin{array}{l}0.78 \\
0.32 \\
0.56\end{array}$ & $\begin{array}{l}0.20 \\
0.00 \\
0.20\end{array}$ & $\begin{array}{l}1.33 \\
0.80 \\
1.07\end{array}$ & $\begin{array}{l}0.43 \\
0.24 \\
0.54\end{array}$ & $\begin{array}{l}0.93 \\
0.36 \\
0.41\end{array}$ & $\begin{array}{l}0.95 \\
0.57 \\
0.80\end{array}$ & $\begin{array}{l}0.14 \\
0.07 \\
0.40\end{array}$ & $\begin{array}{l}0.15 \\
0.07 \\
0.49\end{array}$ & $\begin{array}{l}\overline{0.05} \\
0.18\end{array}$ & $\frac{0.08}{-}$ & $\begin{array}{l}0.15 \\
0.08 \\
0.20\end{array}$ & $\begin{array}{l}0.30 \\
0.13 \\
0.22\end{array}$ \\
\hline- & - & - & - & - & - & - & - & - & - & - & - \\
\hline$\overline{0.21}$ & $\begin{array}{l}0.12 \\
0.03\end{array}$ & $\overline{0.45}$ & $\overline{0.29}$ & $\overline{0.26}$ & $\overline{0.39}$ & $\overline{0.29}$ & $\overline{0.33}$ & $\overline{0.20}$ & $\begin{array}{l}1.06 \\
0.21\end{array}$ & $\begin{array}{l}0.01 \\
0.28\end{array}$ & $\overline{0.18}$ \\
\hline 0.95 & 0.92 & $=$ & $=$ & $=$ & $=$ & $z$ & $\overline{0.07}$ & $=$ & - & $=$ & $\bar{z}$ \\
\hline 0.30 & 0.06 & 0.37 & 0.14 & 0.32 & 0.34 & 0.04 & 0.03 & - & - & 0.01 & - \\
\hline$z$ & $=$ & z & $\bar{z}$ & $\bar{z}$ & $=$ & Z & $=$ & $=$ & $=$ & $=$ & $=$ \\
\hline 1.20 & $=$ & $\overline{0.06}$ & $\overline{0 .(4)}$ & $=$ & $\overline{0.07}$ & $=$ & $\overline{0.07}$ & $\overline{0.05}$ & $=$ & $\overline{0.08}$ & $\overline{0.12}$ \\
\hline $\begin{array}{r}0.19 \\
0.06 \\
19.36\end{array}$ & $\begin{array}{r}0.13 \\
11.85\end{array}$ & $\begin{array}{r}0.99 \\
49.94\end{array}$ & $\begin{array}{r}3.40 \\
2624\end{array}$ & $\overline{0.29}$ & $\begin{array}{r}0.43 \\
34.43\end{array}$ & $\begin{array}{r}0.74 \\
0.900\end{array}$ & $\begin{array}{r}0.38 \\
0.39\end{array}$ & $\begin{array}{l}0.50 \\
808\end{array}$ & $\overline{0.41}$ & $\begin{array}{l}0.28 \\
6.02\end{array}$ & $\begin{array}{l}\overline{0.80} \\
8.04\end{array}$ \\
\hline
\end{tabular}

Hydrolyzable

Relative abundance Total ( $\mu \mathrm{M} / \mathrm{L})$

0 102030405060708090100
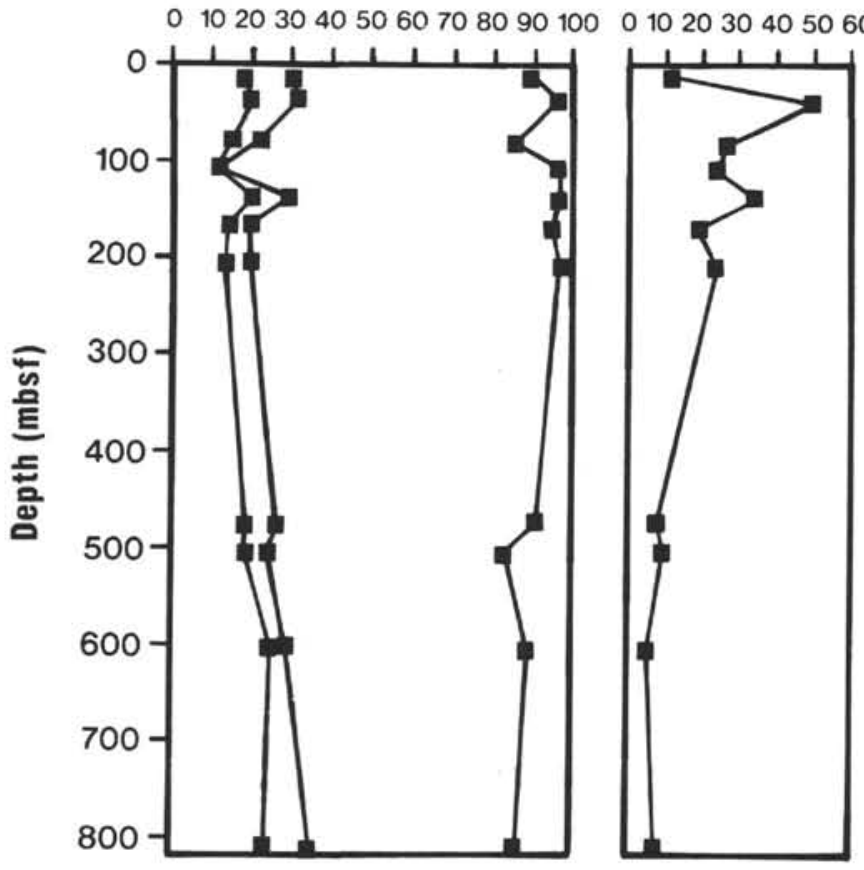

Free

Relative abundance Total $(\mu \mathrm{M} / \mathrm{L})$
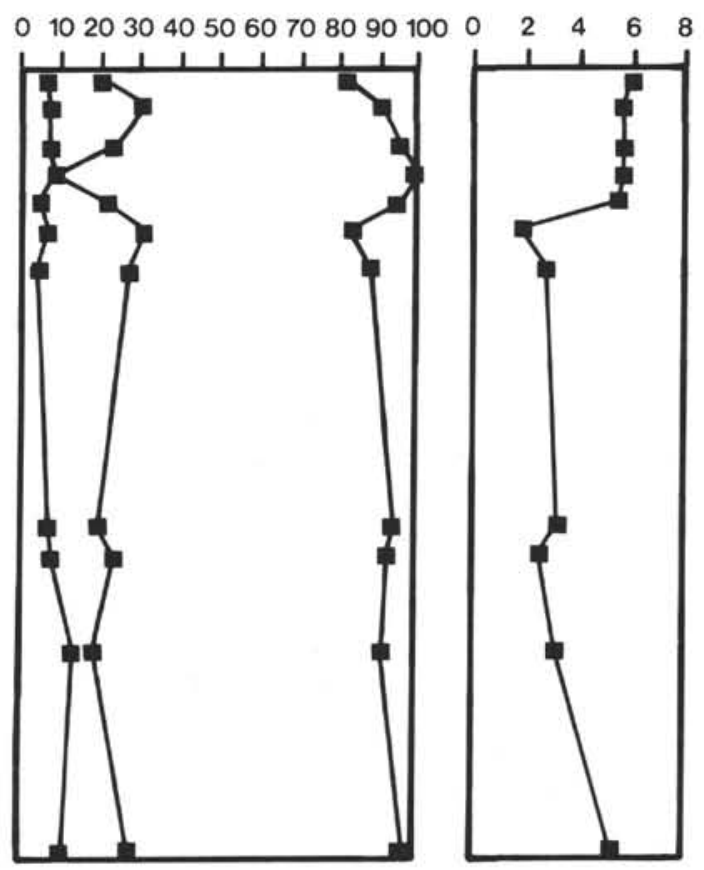

Figure 4. Concentrations of acidic, basic, neutral, and total amino acids of THAA and DFAA in interstitial waters vs. depth, Site 791. 
Table 3. Concentrations in $\mu \mathrm{mol} / \mathrm{L}$ of dissolved free amino acids (DFAA) in interstitial waters, Sites 790 and 791.

\begin{tabular}{|c|c|c|c|c|c|c|c|c|c|c|c|}
\hline \multicolumn{2}{|c|}{$\begin{array}{l}\text { Hole, core, section } \\
\text { Interval }(\mathrm{cm})\end{array}$} & $\begin{array}{c}790 \mathrm{~A}-2 \mathrm{H}-3 \\
140-150\end{array}$ & $\begin{array}{c}790 \mathrm{~A}-3 \mathrm{H}-5 \\
140-150\end{array}$ & $\begin{array}{c}790 \mathrm{~B}-6 \mathrm{H}-3 \\
140-150\end{array}$ & $\begin{array}{c}790 \mathrm{~B}-8 \mathrm{H}-5 \\
140-150\end{array}$ & $\begin{array}{c}790 \mathrm{~B}-9 \mathrm{H}-2 \\
140-150\end{array}$ & $\begin{array}{c}790 \mathrm{C}-2 \mathrm{H}-5 \\
0-5\end{array}$ & $\begin{array}{c}790 \mathrm{C}-5 \mathrm{H}-5 \\
140-150\end{array}$ & $\begin{array}{c}790 \mathrm{C}-10 \mathrm{H}-1 \\
101-111\end{array}$ & $\begin{array}{c}790 \mathrm{C}-13 \mathrm{H}-2 \\
140-150\end{array}$ & $\begin{array}{c}790 \mathrm{C}-16 \mathrm{H}-3 \\
140-150\end{array}$ \\
\hline \multicolumn{2}{|c|}{ Depth (mbsf) } & 13.25 & 25.75 & 46.95 & 69.05 & 74.25 & 100.45 & 130.95 & 165.96 & 196.85 & 227.25 \\
\hline \multicolumn{12}{|l|}{ Acidic } \\
\hline & Glutamic acid & 0.06 & 0.14 & \multicolumn{2}{|c|}{ Basic Glutamic acid } & 0.11 & 0.03 & 0.11 & 0.11 & 0.11 & $\begin{array}{l}0.50 \\
0.19\end{array}$ \\
\hline Dasic & Ornithine & 0.22 & - & 0.36 & 0.23 & 0.38 & 0.22 & 0.35 & 0.13 & 0.32 & 0.80 \\
\hline & Lysine & - & - & - & 0.03 & 0.03 & 0.08 & 0.03 & 0.00 & 0.04 & 0.05 \\
\hline & Histidine & $\overline{0 \times 0}$ & 0.08 & - & - & - & $\overline{0}$ & 0.04 & $\overline{0} e_{3}$ & - & - \\
\hline \multirow{2}{*}{\multicolumn{12}{|c|}{$\begin{array}{l}\text { Neutral } \\
\text { Hydroxy }\end{array}$}} \\
\hline & & & & & & & & & & & \\
\hline & Threonine & 0.18 & 0.11 & 0.09 & 0.16 & 0.10 & 0.18 & 0.34 & 0.08 & 0.15 & 0.41 \\
\hline & Serine & 0.75 & 0.10 & 0.59 & 0.60 & 0.73 & 0.66 & 1.49 & 0.19 & 0.49 & I.11 \\
\hline \multicolumn{12}{|c|}{ Straight } \\
\hline & Alanine & 0.32 & 0.14 & 0.21 & 0.29 & 0.31 & $\begin{array}{l}0.32 \\
0.27\end{array}$ & $\begin{array}{l}1.64 \\
0.70\end{array}$ & $\begin{array}{l}0.23 \\
0.13\end{array}$ & $\begin{array}{l}0.46 \\
0.29\end{array}$ & $\begin{array}{l}1.49 \\
0.85\end{array}$ \\
\hline \multicolumn{12}{|c|}{ Branch } \\
\hline & Valine & $0 .(x)$ & 0.30 & - & 0.26 & 0.13 & 0.23 & - & 0.11 & $\overline{0}$ & - \\
\hline & Iso-leucine & 0.08 & 0.06 & 0.08 & 0.09 & 0.19 & 0.2 .3 & 0.29 & - & 0.07 & 0.24 \\
\hline \multicolumn{12}{|c|}{$\begin{array}{l}\text { Leucine } \\
\text { Other }\end{array}$} \\
\hline \multicolumn{12}{|c|}{$\begin{array}{l}\text { Aspargine } \\
\text { Aromatic }\end{array}$} \\
\hline & Tyrosine & 0.04 & - & - & - & 0.11 & - & 0.13 & 0,05 & - & 0.15 \\
\hline \multirow{3}{*}{ Sulfur } & Phenylalanine & - & 0.02 & - & - & - & - & - & - & - & - \\
\hline & Cystein & 0.12 & - & - & - & - & - & - & - & - & - \\
\hline & Methionine & - & 一 & - & - & - & - & - & - & - & - \\
\hline \multirow{6}{*}{ Other } & Taurine & $0 .(x)$ & 0.20 & - & 0.0 .3 & - & 0.02 & - & - & - & - \\
\hline & Citrulline & - & 0.08 & - & - & - & - & - & - & - & - \\
\hline & $\alpha$-aminoadipic acid & - & - & - & - & - & - & - & - & - & - \\
\hline & $\alpha$-aminobutyric acid & - & - & - & - & - & - & - & - & - & - \\
\hline & $\beta$-aminobutyric acid & - & - & - & - & - & - & - & - & - & - \\
\hline & $\begin{array}{l}\gamma \text {-aminobutyric acid } \\
\beta \text {-alanine }\end{array}$ & - & - & $\overline{004}$ & $=$ & - & - & $\overline{003}$ & $\overline{0(x)}$ & - & - \\
\hline Total & 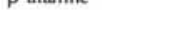 & 2.88 & 1.59 & 2.28 & 2.70 & 3.19 & $2.6 \%$ & 5.76 & 1.26 & 2.31 & 6.01 \\
\hline
\end{tabular}

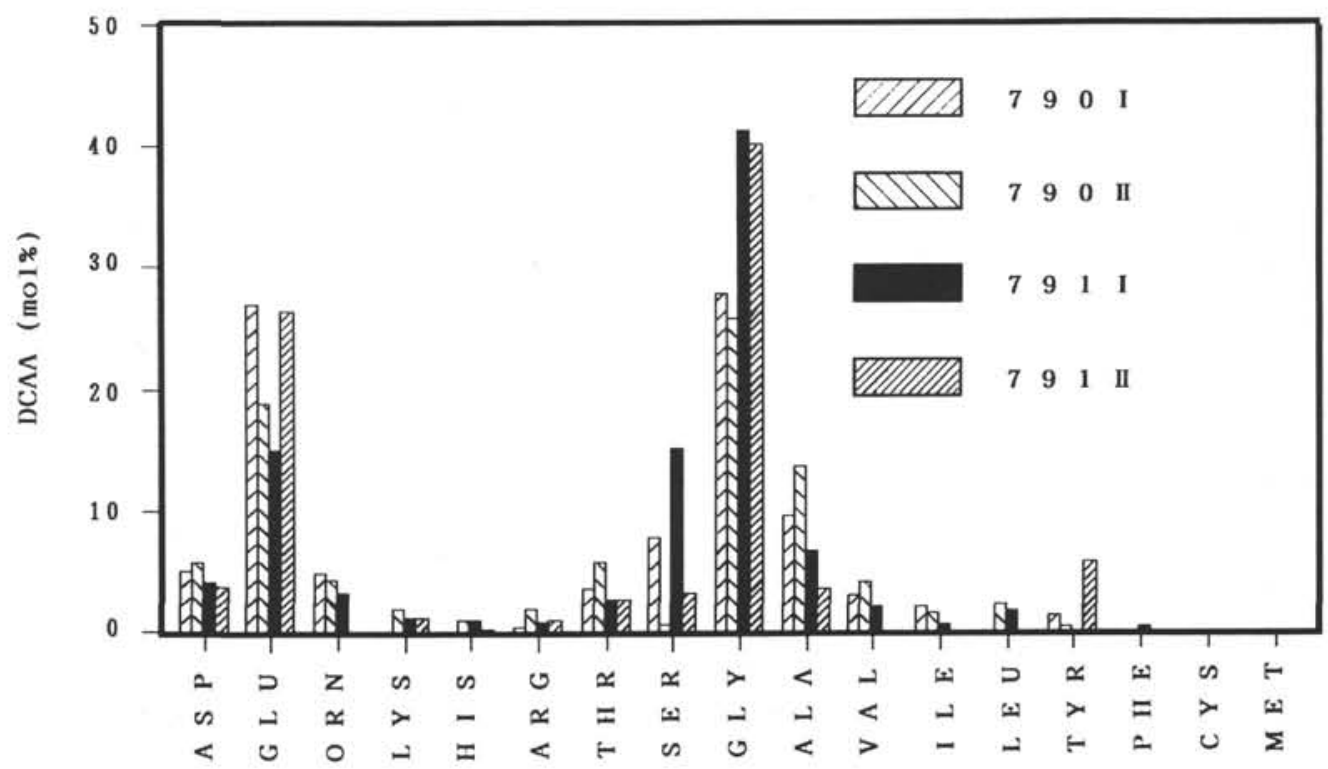

Figure 5. Composition of DCAA in interstitial waters, Sites 790 and 791. The mean mole percentage of each amino acid from all lithologic units in both sites is plotted. See Figure 3 for an explanation of abbreviations.

(Siegel and Degens, 1966; Kawahata and Ishizuka, 1989; Lee and Bada, 1975). The mean values and relative abundances of acidic, basic, neutral, aromatic, and sulfur-containing amino acids are 2.29 (22 $\mathrm{mol} \%), 0.60$ (6 mol\%), 6.70 (65 mol\%), 0.09 (1 mol\%), and 0.00 $\mu \mathrm{mol} / \mathrm{L}(0 \mathrm{~mol} \%)$, respectively.

Smear slide observations indicate that the major constituents of the sediments from Sites 790 and 791 are volcanic glass, feldspar, clays, and calcareous nannofossils. The high sedimentation rate at Sites 790 and 791 is a result of the large input of volcanic glass to the sediments. Glass and feldspar lack organic material. The geographic and geologic situation of both sites eliminates the possibility of a major contribution of land-derived amino acids. Thus, the amino acids in the interstitial waters originate from those of calcareous biogenic debris, with lesser amounts of siliceous debris.

One of the important characteristics of the relationship between the amino acids of the source material and the DCAA is that aspartic acid and the basic fraction, except for ornithine, are much more depleted in DCAA (Figs. 5 and 6 and Table 5). On the other hand, the characteristics of the amino acids in the interstitial waters indicate a greater depletion of the acidic amino acid fraction in the DFAA than in the 
Table 3 (continued).

\begin{tabular}{|c|c|c|c|c|c|c|c|c|c|c|c|}
\hline $\begin{array}{c}790 \mathrm{C}-18 \mathrm{H}-2 \\
140-150\end{array}$ & $\begin{array}{l}791 \mathrm{~A}-2 \mathrm{H}-5 \\
140-150\end{array}$ & $\begin{array}{l}791 \mathrm{~A}-5 \mathrm{H}-3 \\
140-150\end{array}$ & $\begin{array}{c}791 \mathrm{~A}-9 \mathrm{H}-6 \\
140-150\end{array}$ & $\begin{array}{l}791 \mathrm{~A}-12 \mathrm{H}-5 \\
140-150\end{array}$ & $\begin{array}{c}791 \mathrm{~A}-15 \mathrm{H}-6 \\
25-35\end{array}$ & $\begin{array}{c}791 \mathrm{~A}-18 \mathrm{H}-6 \\
90-100\end{array}$ & $\begin{array}{c}791 \mathrm{~A}-22 \mathrm{H}-6 \\
140-150\end{array}$ & $\begin{array}{l}791 \mathrm{~B}-10 \mathrm{R}-1 \\
86-96\end{array}$ & $\begin{array}{c}791 \mathrm{~B}-13 \mathrm{R}-1 \\
140-150\end{array}$ & $\begin{array}{l}791 \mathrm{~B}-23 \mathrm{R}-3 \\
115-125\end{array}$ & $\begin{array}{c}791 B-45 R-2 \\
140-150\end{array}$ \\
\hline 254.65 & 11.95 & 37,45 & 80.25 & 107,85 & 137.30 & 166.95 & 205.85 & 474.21 & 503.55 & 602.90 & 813.20 \\
\hline 0.29 & 0.29 & 0.30 & 0.25 & 0.32 & 0.24 & 0.09 & 0.12 & 0.19 & 0.17 & 0.27 & 0.37 \\
\hline 0.19 & 0.11 & 0.10 & 0.15 & 0.13 & - & 0.04 & 0.00 & 0.05 & 0.04 & 0.16 & 0.24 \\
\hline 0.45 & 0.62 & 0.81 & 0.45 & - & 0.49 & 0.26 & 0.32 & 0.19 & 0.24 & 0.03 & 0.57 \\
\hline 0.16 & 0.18 & 0.18 & 0.24 & - & 0.15 & $0 .(0)$ & 0.16 & 0.10 & $0 .(x)$ & 0.11 & 0.16 \\
\hline 0.27 & - & 0.32 & 0.19 & - & 0.22 & 0.16 & 0.18 & 0.09 & 0.13 & - & 0.13 \\
\hline 0.04 & - & - & - & - & - & $0 .(0)$ & 0.07 & 0.01 & - & - & - \\
\hline 0.29 & 0.30 & 0.29 & 0.32 & 0.40 & 0.27 & $0 .(k)$ & 0.14 & 0.18 & 0.13 & 0.19 & 0.20 \\
\hline 1.29 & 1.38 & 1.46 & 1.33 & 1.78 & 1.26 & 0.24 & 0.46 & 0.73 & 0.49 & 0.39 & 0.74 \\
\hline 1.14 & 1.04 & 0.97 & 1.04 & 1.38 & $1 .(19)$ & 0.23 & 0.48 & 0.76 & 0.62 & 0.68 & 1.34 \\
\hline 0.72 & 0.54 & - & 0.54 & 0.65 & 0.54 & 0.15 & 0.25 & 0.45 & 0.36 & 0.54 & 0.74 \\
\hline 0.21 & 0.11 & 0.15 & 0.25 & 0.23 & 0.22 & - & 0.12 & - & 0.08 & 0.15 & 0.30 \\
\hline 0.32 & - & 0.28 & 0.24 & 0.31 & 0.33 & $\overline{0.01}$ & 0.07 & 0.05 & 0.00 & 0.08 & 0.13 \\
\hline 0.27 & 0.19 & 0.17 & 0.22 & 0.25 & 0.25 & 0.17 & 0.15 & 0.18 & - & 0.20 & 0.22 \\
\hline- & - & - & - & - & - & - & - & - & - & - & - \\
\hline- & 0.10 & - & - & - & - & - & - & - & - & 0.01 & - \\
\hline$\overline{0.21}$ & 0.03 & $\overline{0.45}$ & $\overline{0.21}$ & $\overline{0.04}$ & $\overline{0.24}$ & $\overline{0.29}$ & $\overline{0.33}$ & $\overline{0.20}$ & $\overline{0.21}$ & 0.28 & 0.18 \\
\hline 0.95 & 0.92 & - & - & - & - & - & - & - & - & - & - \\
\hline- & - & - & - & - & - & - & - & - & - & - & - \\
\hline 0.03 & 0.01 & - & 0.07 & - & 0.04 & 0.01 & - & - & - & - & - \\
\hline- & - & - & - & - & - & Wor & - & - & - & - & - \\
\hline- & - & - & - & - & - & - & - & - & - & - & - \\
\hline$=$ & E & - & - & - & - & - & - & - & - & $=$ & $=$ \\
\hline 二 & Z & - & $=$ & 二 & E & 三 & $=$ & Z & - & $=$ & $\bar{z}$ \\
\hline$\overline{682}$ & - & 0.10 & - & - & - & 二 & 0.01 & - & $=$ & $=$ & $=$ \\
\hline 6.82 & 5.83 & 5.59 & 5.52 & 5,49 & 5.35 & 1.74 & 2.75 & 3.17 & 2.47 & 3.09 & 5.32 \\
\hline
\end{tabular}

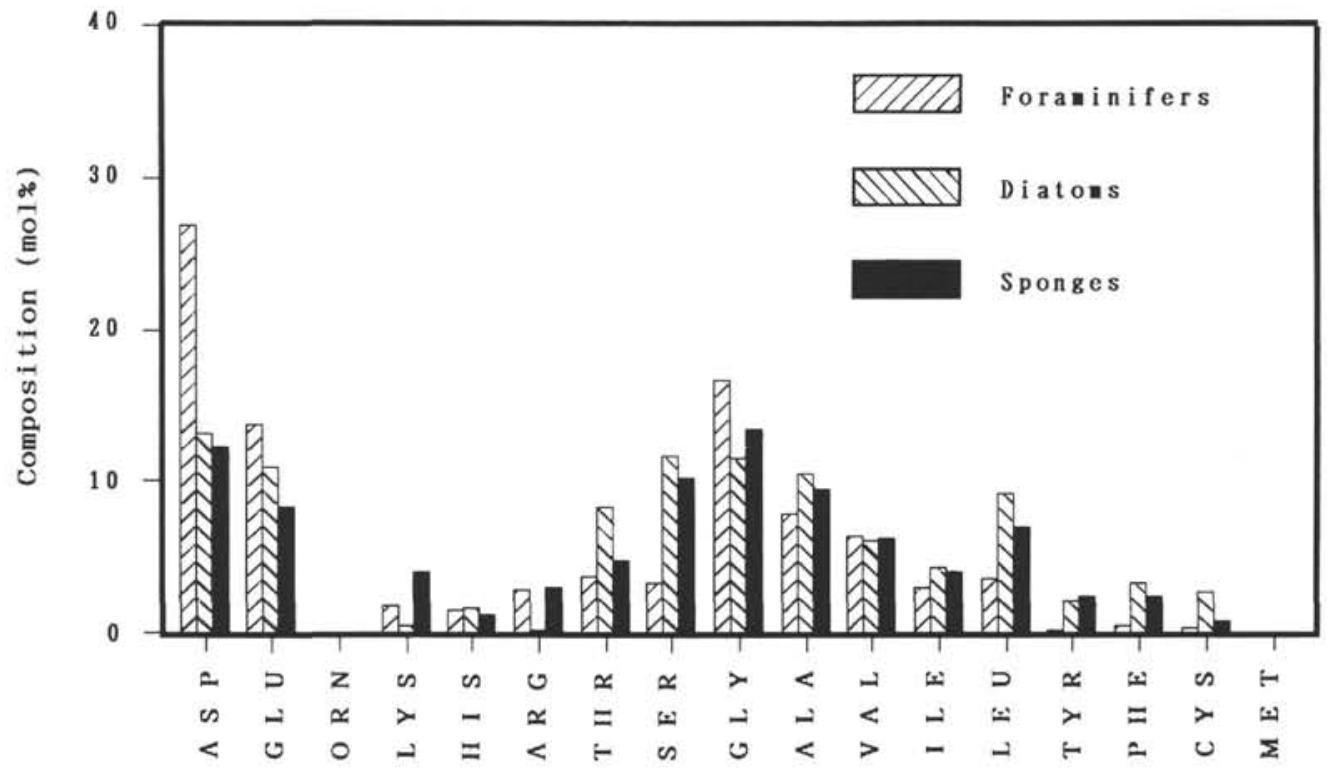

Figure 6. Amino acid composition of foraminifers, diatoms, and sponges plotted for comparison with DCAA and DFAA. See Figure 3 for an explanation of abbreviations.

DCAA and an enrichment of glycine and serine in both. The adsorption or reaction of the amino acids in the interstitial waters with biogenic carbonates would be responsible for the lower relative abundance of the acidic fraction of the DFAA than the DCAA (Kawahata and Ishizuka, 1989). Glycine is possibly derived from serine, threonine, valine, tyrosine, and histidine by decomposition from heating (Terashima and Inouchi, 1990). In addition, it has the simplest structure of the protein amino acids and a greater advantage in its stability in solution than the other amino acids that have a more complicated structure, which would raise its relative abundance in interstitial waters.

\section{Biogeochemical Characteristics of Minor Constituent Amino Acids in Interstitial Waters}

Information on the nature of the amino acids in the interstitial waters can be obtained from the distribution of minor constituents. Especially significant in this regard is the presence of nonprotein amino acids, about $10 \mathrm{~mol} \%$ of the total DCAA.

The mean relative abundances of $\beta$-alanine and $\gamma$ amino butyric amino acid are about 4 and $1 \mathrm{~mol} \%$ of the total DCAA at Sites 790 and 791, respectively, whereas neither is found in DFAA. Although 
Table 4. Concentrations in $\mu \mathrm{mol} / \mathrm{L}$ of dissolved combined amino acids (DCAA) in interstitial waters, Sites 790 and 791.

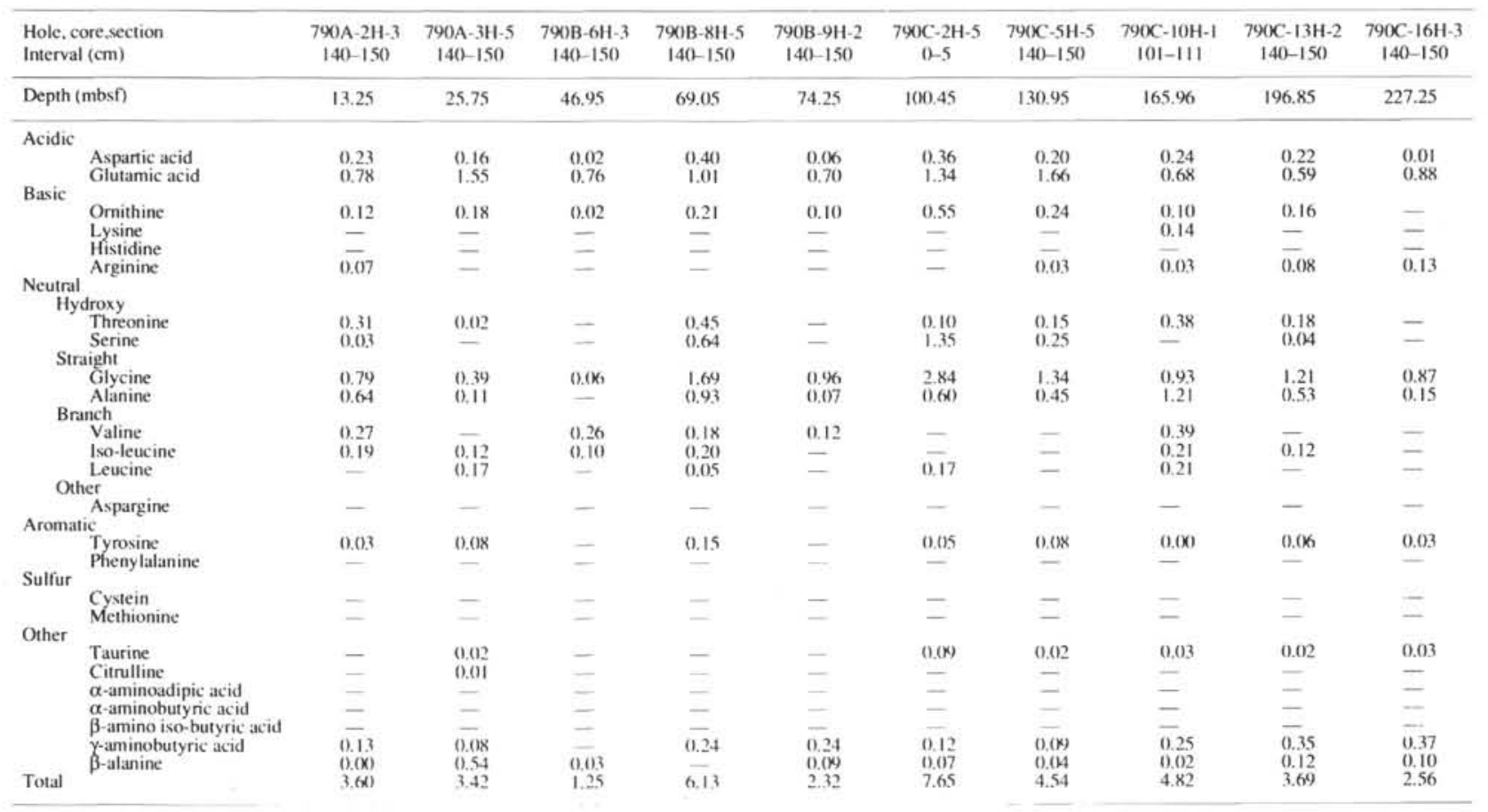

$\beta$-alanine and $\gamma$ amino butyric amino acid are generally thought to be decomposition products of aspartic and glutamic acids, respectively, there was no evidence for their production during studies on either ultracleaned foraminifer tests or various proteins in buffered solution (Schroeder, 1975). Thus, it appears that their common presence in sediments from the natural environment is related to the enzymatic decomposition of aspartic and glutamic acids (Ittekkot et al., 1984b). Such decomposition can take place either on particles (Lee and Cronin, 1982) or in the guts of organisms (Ittekot et al., 1984b). Jannasch et al. (1971) suggested that microbial degradation is slow in the deep-sea environment, and Wafer et al. (1982) showed that most of the degradation of polypeptides takes place at depths above $1000 \mathrm{~m}$ and that flux changes below this depth are much slower. However, in general the relative abundance of nonprotein amino acids collected in sediment traps is fairly low ( $<3 \mathrm{~mol} \%$ ) (Ittekkot et al., 1984a, 1984b). Thus, the major production of nonprotein amino acids probably occurred below the sediment/seawater interface. The concentrations of $\beta$-alanine and $\gamma$ amino butyric acid observed are the net result of processes that release and remove to and from the interstitial waters. The absence of $\beta$-alanine and $\gamma$-amino butyric acid in the DFAA suggests that these two amino acids are very slowly released to the DFAA reservoir, or, rather, are decomposed rapidly by unstable species in the interstitial waters.

Ornithine is also an important nonprotein amino acid. It is absent from the foraminifer, diatom, and sponge tests (Degens, 1970) and is much depleted in marine particulate matter (Ittekkot et al., 1984a). The mean relative abundance of ornithine in the DCAA and DFAA from Sites 790 and 791 is about 3 and 9 mol\%, respectively. The molar ratio of ornithine to the other basic amino acids (lysine, histidine, and arginine) is 1.2 in DCAA and 2.1 in DFAA. The basic amino acid fraction is less stable than the acidic fraction in the free state. In adsorption experiments of the amino acids in distilled water, basic (positively charged) amino acids are strongly adsorbed (40\%-80\% removal) and neutral (uncharged) amino acids were taken up appreciably $(10 \%-15 \%)$ by montmorillonite. Montmorillonite is an alteration product of volcanic glass and seawater that is present in small to appreciable amounts in Sites 790 and 791 . Thus, the basic amino acid fraction tends to be decomposed in the interstitial waters and/or incorporated into the lattice of clay minerals, which would result in a decrease in the relative abundance of the basic amino acids. On the other hand, ornithine is a decomposed product of arginine. The relative abundance of arginine in the DCAA and DFAA of Sites 790 and 791 is much less than those of marine particulate matter and biogenic debris, which supports the hypothesis that ornithine can be produced during early diagenesis.

\section{ACKNOWLEDGMENTS}

The authors wish to acknowledge Dr. K. Fujioka, co-chief scientist of Leg 126, for giving us the opportunity to study the amino acids in interstitial waters from the Izu-Bonin island arcs, Drs. I. Koike and M. Ohmori for providing technical advice on analytical procedures, and reviewers for improving our manuscript. Also, we are grateful for the shipboard technicians of the Ocean Drilling Program for squeezing the interstitial waters from the sediments. This work was supported by research grants from the Agency of Industrial Science, Science and Technology Agency of Japan, and Technology and the Ministry of Education.

\section{REFERENCES}

Christensen, D., and Blackburn, T. H., 1980. Turnover of tracer $\left({ }^{14} \mathrm{C},{ }^{3} \mathrm{H}\right.$ labelled) alanine in shore marine sediments. Mar. Biol., 58:97-103.

Degens, E. T., 1970. Molecular nature of nitrogenous compounds in seawater and recent sediments. In Hood, D. W. (Ed.), Organic Matter in Natural Water. Univ. Alaska Inst. Mar. Sci., Occas. Publ., 1:77-106.

Henrichs, S. M., and Farrington, J. W., 1979. Amino acids in interstitial waters of marine sediments. Nature, 279:319-322.

Henrichs, S. M., Farrington, J. W., and Lee, C., 1984. Peru upwelling region sediments near $15^{\circ} \mathrm{S} .2$. Dissolved free and total hydrolyzable amino acids. Limnol. Oceanogr., 29:20-34.

Ishizuka, T., Ittekkot, V., and Kawahata, H., 1990. Geochemistry of amino acids in interstitial water, Leg 116. In Cochran, J. R., Stow, D.A.V., et al., Proc. ODP, Sci. Results, 116: College Station, TX(Ocean Drilling Program), 141-144.

Ishizuka, T., Nozaki, Y., and Shimooka, K., 1988. Amino acids in the interstitial waters of ESOPE long cores from two North Atlantic abyssal plains. Geochem. J., 22:1-8. 
Table 4 (continued).

\begin{tabular}{|c|c|c|c|c|c|c|c|c|c|c|c|}
\hline $\begin{array}{c}790 \mathrm{C}-18 \mathrm{H}-2 \\
140-150\end{array}$ & $\begin{array}{c}791 \mathrm{~A}-2 \mathrm{H}-5 \\
140-150\end{array}$ & $\begin{array}{c}791 \mathrm{~A}-5 \mathrm{H}-3 \\
140-150\end{array}$ & $\begin{array}{c}791 \mathrm{~A}-9 \mathrm{H}-6 \\
140-150\end{array}$ & $\begin{array}{c}791 \mathrm{~A}-12 \mathrm{H}-5 \\
140-150\end{array}$ & $\begin{array}{c}791 \mathrm{~A}-15 \mathrm{H}-6 \\
25-35\end{array}$ & $\begin{array}{c}791 \mathrm{~A}-18 \mathrm{H}-6 \\
9(0-1(\mathrm{~K})\end{array}$ & $\begin{array}{c}791 \mathrm{~A}-22 \mathrm{H}-6 \\
140-150\end{array}$ & $\begin{array}{c}791 \mathrm{~B}-10 \mathrm{R}-1 \\
86-96\end{array}$ & $\begin{array}{c}791 \mathrm{~B}-1,3 \mathrm{R}-1 \\
140-150\end{array}$ & $\begin{array}{c}791 \mathrm{~B}-23 \mathrm{R}-3 \\
115-125\end{array}$ & $\begin{array}{c}791 \mathrm{~B}-45 \mathrm{R}-2 \\
140-150\end{array}$ \\
\hline 254.65 & 11.95 & 37.45 & 80.25 & 107.85 & 137.30 & 166.95 & 205.85 & 474.21 & 503.55 & 602.90 & 813.20 \\
\hline $\begin{array}{l}0.87 \\
2.31\end{array}$ & $\begin{array}{l}0.28 \\
1.38\end{array}$ & $\begin{array}{l}1.78 \\
8.03\end{array}$ & $\begin{array}{l}0.75 \\
2.6 \mathrm{~K}\end{array}$ & $\begin{array}{l}0.52 \\
1.74\end{array}$ & $\begin{array}{l}1.50 \\
5.38\end{array}$ & $\begin{array}{l}0.73 \\
1.89\end{array}$ & $\begin{array}{l}0.93 \\
2.27\end{array}$ & $\begin{array}{l}0.14 \\
1.18\end{array}$ & $\begin{array}{l}0.36 \\
1.33\end{array}$ & $\begin{array}{l}0.13 \\
1.00\end{array}$ & $\begin{array}{l}0.03 \\
1.33\end{array}$ \\
\hline $\begin{array}{l}0.73 \\
0.29 \\
0.22 \\
0.20\end{array}$ & $\begin{array}{l}0.21 \\
0.12 \\
0.24 \\
0.12\end{array}$ & $\begin{array}{l}2.65 \\
0.57 \\
0.69 \\
0.31\end{array}$ & $\begin{array}{l}0.51 \\
0.14 \\
\overline{0.25}\end{array}$ & $\underline{z}$ & $\begin{array}{l}1.54 \\
0.31 \\
0.43 \\
-\end{array}$ & $\begin{array}{l}\overline{0.24} \\
0.04 \\
0.18\end{array}$ & $\begin{array}{l}0.62 \\
0.31 \\
0.13 \\
0.26\end{array}$ & $\begin{array}{l}\overline{0.02} \\
\overline{0.18}\end{array}$ & $\begin{array}{l}\overline{0.17} \\
0.03\end{array}$ & $\begin{array}{l}\overline{0.02} \\
- \\
-\end{array}$ & $=$ \\
\hline $\begin{array}{l}0.78 \\
0.11\end{array}$ & $\begin{array}{l}0.12 \\
2.22\end{array}$ & $\begin{array}{l}1.48 \\
7.21\end{array}$ & $\begin{array}{l}0.29 \\
1.92\end{array}$ & $\begin{array}{l}0.98 \\
4.56\end{array}$ & $\begin{array}{l}0.74 \\
3.74\end{array}$ & $\begin{array}{l}0.26 \\
1.86\end{array}$ & $\begin{array}{l}0.29 \\
2.29\end{array}$ & 0.06 & $\begin{array}{l}0.29 \\
0.59\end{array}$ & 0.12 & 二 \\
\hline $\begin{array}{l}3.08 \\
1.35\end{array}$ & $\begin{array}{l}0.88 \\
0.11\end{array}$ & $\begin{array}{r}13.94 \\
3.79\end{array}$ & $\begin{array}{l}9.08 \\
0.97\end{array}$ & $\begin{array}{l}6.79 \\
2.21\end{array}$ & $\begin{array}{r}10.93 \\
2.02\end{array}$ & $\begin{array}{r}10.22 \\
0.66\end{array}$ & $\begin{array}{r}13.00 \\
0.75\end{array}$ & $\begin{array}{l}2.70 \\
0.07\end{array}$ & $\begin{array}{l}3.20 \\
0.32\end{array}$ & $\begin{array}{l}1.04 \\
0.23\end{array}$ & 0.43 \\
\hline $\begin{array}{l}0.57 \\
0.01 \\
0.30\end{array}$ & $\frac{0 .(k)}{0.03}$ & $\begin{array}{l}1.18 \\
0.53 \\
0.89\end{array}$ & $\frac{0.18}{0.32}$ & $\begin{array}{l}0.70 \\
0.05 \\
0.16\end{array}$ & $\begin{array}{l}0.73 \\
0.24 \\
0.55\end{array}$ & $\begin{array}{l}0.14 \\
0.06 \\
0.23\end{array}$ & $\begin{array}{l}0.03 \\
0.01 \\
0.35\end{array}$ & $=$ & $\bar{z}$ & E & $=$ \\
\hline$=$ & 0.03 & - & $\overline{0.09}$ & $\overline{0.22}$ & $\overline{0.15}$ & $=$ & $\overline{-}$ & $=$ & 1.06 & 二 & $=$ \\
\hline$\underline{-}$ & $\overline{-}$ & - & - & - & $=$ & - & $\overline{0.07}$ & $=$ & $=$ & - & $=$ \\
\hline 0.27 & 0.14 & 0.37 & 0.06 & 0.32 & 0.31 & 0.02 & 0.03 & - & - & 0.01 & - \\
\hline - & - & - & - & - & - & - & - & - & - & - & - \\
\hline$\overline{1.20}$ & 二 & E & E & E & 二 & 二 & E & $\bar{z}$ & $E$ & $=$ & $=$ \\
\hline$\overline{0}$ & $\overline{10}$ & 0.06 & $0 .(x)$ & - & 0.07 & - & 0.07 & 0.05 & - & 0.08 & 0.12 \\
\hline $\begin{array}{l}0.19 \\
0.06\end{array}$ & 0.13 & $\overline{0.89}$ & 340 & $\overline{0} 20$ & $\overline{043}$ & $\overline{074}$ & & & & & 080 \\
\hline $\begin{array}{l}0.00 \\
12.55\end{array}$ & $\overline{6.02}$ & $\begin{array}{r}0.89 \\
44.35\end{array}$ & 20.73 & $\begin{array}{r}0.29 \\
18.52\end{array}$ & $\begin{array}{r}0.43 \\
29.08\end{array}$ & $\begin{array}{r}0.74 \\
17.28\end{array}$ & $\begin{array}{r}0.37 \\
21.18\end{array}$ & 4.91 & $\begin{array}{l}0.41 \\
7.77\end{array}$ & $\begin{array}{l}0.28 \\
2.93\end{array}$ & $\begin{array}{l}0.80 \\
2.72\end{array}$ \\
\hline
\end{tabular}

Ittekkot, V., Degens, E. T., and Honjo, S., 1984a. Seasonality in the fluxes of sugars, amino acids, and amino sugars to the deep ocean: Panama Basin. Deep-sea Res., Pt. A, 31:1071-1083.

Ittekkot, V., Deuser, W. G., and Degens, E. T., 1984b. Seasonality in the fluxes of sugars, amino acids, and amino sugars to the deep ocean: Sargasso Sea. Deep-sea Res., Pt. A, 31:1057-1069.

Jannasch, H. W., Elmhjellen, C. O., Wirsen, C. O., and Farmanfarmaian, A. 1971. Microbial degradation of organic matter in the deep sea. Science, 171:672-675.

Jørgensen, N.O.G., Lindroth, P., and Mopper, K., 1981. Extraction and distribution of free amino acids and ammonium in sediments and overlying sea waters from the Limfjord, Denmark. Oceanol. Acta, 4:465-474.

Kawahata, H., and Ishizuka, T., 1989. Organic properties of sediments and amino acids in interstitial waters from the flank of the Costa Rica Rift, Galapagos spreading center (ODP Sites 677 and 678). In Becker, K., Sakai, H., et al.,Proc. ODP, Sci. Results, 111: College Station, TX (Ocean Drilling Program), 215-225.

Kawahata, H., Ishizuka, T., and Nagao, T., 1990. Amino acids in the interstitial waters from ODP Site 695 in the Weddell Sea, Antarctic Ocean. In Barker, P. F., Kennett, J. P., et al., Proc. ODP, Sci. Results, 113: College Station, TX (Ocean Drilling Program), 179-187.

King, J. J., 1974. Preserved amino acids from silicified protein in fossil radiolarians. Nature, 252:690-692.

Lee, C., and Bada, J. L., 1975. Amino acids in equatorial Pacific Ocean water. Earth Planet. Sci. Lett., 26:61-68.

Lee, C., and Cronin, C., 1982. The vertical flux of particulate organic nitrogen in the sea: decomposition of amino acids in the Peru upwelling area and the equatorial Atlantic. J. Mar. Res., 40:227-251.

Malta, Y., Montana, S., and Ishii, J., 1982. Early diagenesis of amino acids in Okhotsk Sea sediments. Deep-sea Res., Pt. A, 29:485-498.

Peake, E. P., Baker, B. L., and Hodgson, G. W., 1972. Hydrogeochemistry of the surface waters of the Mackenzie River drainage basin, Canada. II. The contribution of amino acids, hydrocarbons and chlorine to the Beaufort Sea by the Mackenzie River system. Geochim. Cosmochim. Acta, 36:867-883.

Rosenfeld, J. K., 1979. Amino acid diagenesis and adsorption in nearshore anoxic sediments. Limnol. Oceanogr., 24:1014-1021.

Shipboard Scientific Party, 1990. Sites 790/791. In Taylor, B., Fujioka, K., et al., Proc. ODP, Init. Repts., 126: College Station, TX (Ocean Drilling Program), 127-221.

Shroeder, R. A., 1975. Absence of $\beta$-alanine and $\gamma$ aminobutyric acid in cleaned foraminiferal shells: implications for use as a chemical criterion to indicate removal of nonindigenous amino acid contaminants. Earth Planet. Sci. Lett., 25:272-278.

Siegel, A., and Degens, E. T., 1966. Concentration of dissolved amino acids from saline waters by ligand-exchange chromatography. Science, 151:1098.

Terashima, M., and Inouchi, Y., 1990. Geochemistry of amino acids and monosaccharides in bottom sediments from Lake Kasumigaura, Central Japan. Bull. Geol. Soc. Jpn., 41:641-656.

Wefer, G., Suess, E., Balzer, W., Liebezeit, G., Müller, P. J., Ungerer, A., and Wenk, W., 1982. Fluxes of biogenic components from sediment trap deployment in circumpolar waters of Drake Passage. Nature, 299:145-147.

Whelan, J. K., 1977. Amino acids in a surface sediment core of the Atlantic abyssal plain. Geochim. Cosmochim. Acta, 41:803-810.

Date of initial receipt: 2 January 1991

Date of acceptance: 19 August 1991

Ms 126B-154 
Table 5. Amino acid composition of interstitial waters from Sites 790 and 791, particulate matter, and marine plankton.

\begin{tabular}{|c|c|c|c|c|c|c|c|c|c|c|c|c|c|c|c|}
\hline & & \multicolumn{8}{|c|}{ Intertitial waters } & \multirow{3}{*}{\multicolumn{2}{|c|}{ Particulate marter }} & \multirow{4}{*}{$\begin{array}{l}\text { Foraminifers } \\
\text { (molf) }\end{array}$} & \multirow{4}{*}{$\begin{array}{l}\text { Diatoms } \\
\text { (mol/\%) }\end{array}$} & \multirow{4}{*}{$\begin{array}{l}\text { Sponges } \\
\text { (mol\%) }\end{array}$} & \multirow{4}{*}{ 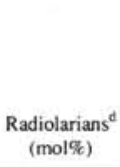 } \\
\hline & & \multicolumn{4}{|c|}{ DFAA } & \multicolumn{4}{|c|}{ DCAA } & & & & & & \\
\hline & & \multicolumn{2}{|c|}{ Site 790} & \multicolumn{2}{|c|}{ Site 791} & \multicolumn{2}{|c|}{ Site 790} & \multicolumn{2}{|c|}{ Site 791} & & & & & & \\
\hline & & $(\mu \mathrm{M})$ & $(\mathrm{mol} \%)$ & $(\mu \mathrm{M})$ & $\left(\mathrm{mol}^{2} / \mathrm{t}\right)$ & $(\mu \mathrm{M})$ & $\left(\mathrm{mol}^{2} / k\right)$ & $(\mu \mathrm{M})$ & $(\mathrm{mol} \%)$ & Marine $^{4}$ & River" & & & & \\
\hline Acidic & & & 9.3 & & 7.8 & & 28.6 & & 20.2 & 25.3 & 24,9 & 40.7 & 24.1 & 20.6 & 28.3 \\
\hline & $\begin{array}{l}\text { Aspartic acid } \\
\text { Glutamic acid }\end{array}$ & $\begin{array}{l}0.22 \\
0.10\end{array}$ & $\begin{array}{l}6.5 \\
2.8\end{array}$ & $\begin{array}{l}0.24 \\
0.09\end{array}$ & 5.6 & 0.25 & 25.3 & 0.65 & 4.1 & $\begin{array}{l}16.7 \\
86\end{array}$ & 13.1 & 26.9 & 13.2 & 12.3 & 15.6 \\
\hline Basic & Glutamic acid & 0.10 & 11.8 & 0.09 & $1 \frac{2}{4} .4$ & 1.11 & 6.8 & 2.57 & $\begin{array}{r}16.1 \\
5.5\end{array}$ & $\begin{array}{c}8.6 \\
14.5\end{array}$ & 3.0 & $\begin{array}{r}13.8 \\
6.0\end{array}$ & $\begin{array}{r}10.9 \\
2.3\end{array}$ & $\begin{array}{l}8.3 \\
8.2\end{array}$ & 8.3 \\
\hline & $\begin{array}{l}\text { Ornithine } \\
\text { Lysine }\end{array}$ & $\begin{array}{l}0.31 \\
0.04\end{array}$ & 9.2 & $\begin{array}{l}0.36 \\
0.11\end{array}$ & $\begin{array}{l}8.6 \\
26\end{array}$ & 0.22 & ${ }_{0.6}^{4.6}$ & 0.45 & 2.8 & $\begin{array}{l}0.1 \\
8.6\end{array}$ & 0.9 & $\overline{17}$ & $\overline{0.5}$ & $\overline{4.0}$ & $\begin{array}{l}0.9 \\
3.9\end{array}$ \\
\hline & Histidine & 0.04 & 1. 10 & 0.13 & 3.1 & 0.02 & 0.4 & 0.14 & 0.9 & $\begin{array}{l}8.0 \\
1.9\end{array}$ & -1 & 1.5 & 1.6 & 1.2 & 0.2 \\
\hline & Arginine & 0.01 & 0.2 & & 02 & 0.5 & 1.0 & 0.12 & 0.7 & 3.9 & - & 2.8 & 0.2 & 3.0 & 3.3 \\
\hline $\begin{array}{c}\text { Neutral } \\
\text { Hyd }\end{array}$ & droxy & & 73.3 & & 69.7 & - & 54.6 & & 67.8 & 51.6 & 61.2 & 44.2 & 61.2 & 54.9 & 58.1 \\
\hline & $\begin{array}{l}\text { Threonine } \\
\text { Serine }\end{array}$ & 0.19 & 2.6 .6 & 0.23 & 5.4 & 0.21 & 4.5 & 0.42 & 13.6 & 5.0 & 4.0 & 3.7 & 8.2 & 4.7 & 4.4 \\
\hline & $\begin{array}{l}\text { Serine } \\
\text { night }\end{array}$ & 0.73 & & & 22.1 & & & 2.22 & 13.9 & 7.2 & 6.7 & 3.2 & 11.6 & 10.2 & 6.3 \\
\hline & $\begin{array}{l}\text { Glycine } \\
\text { Alanine }\end{array}$ & $\begin{array}{l}0.78 \\
0.38\end{array}$ & $\begin{array}{l}22.8 \\
11.3\end{array}$ & $\begin{array}{l}0.88 \\
0.43\end{array}$ & $\begin{array}{l}20.8 \\
10.3\end{array}$ & $\begin{array}{l}1.29 \\
0.55\end{array}$ & $\begin{array}{l}27.1 \\
11.5\end{array}$ & $\begin{array}{l}6.56 \\
1.01\end{array}$ & $\begin{array}{r}41.2 \\
6.4\end{array}$ & $\begin{array}{r}17.9 \\
9.6\end{array}$ & $\begin{array}{l}18.3 \\
14.8\end{array}$ & $\begin{array}{r}16.7 \\
7.8\end{array}$ & $\begin{array}{l}11.5 \\
10.5\end{array}$ & $\begin{array}{r}13.4 \\
9.4\end{array}$ & $\begin{array}{r}22.7 \\
9.0\end{array}$ \\
\hline & $\begin{array}{l}\text { Inch } \\
\text { Valine }\end{array}$ & & 3.6 & & & & 3.4 & & 1.7 & 5.0 & 6.2 & 6.3 & 6.0 & 6.2 & 7.1 \\
\hline & Iso-leucine & 0.15 & 4.4 & 0.14 & 3.2 & 0.09 & 1.8 & 0.08 & 0.5 & 2.5 & 3.0 & 3.0 & 4.3 & 4.0 & 2.7 \\
\hline Oth & $\begin{array}{l}\text { Leeucine } \\
\text { Ler }\end{array}$ & 0.15 & 4.3 & 0.18 & 4.3 & 0.08 & 1.7 & 0.23 & 1.4 & 4.4 & 8.3 & 3.5 & 9.1 & 7.0 & 5.9 \\
\hline Aromati & $\begin{array}{l}\text { Aspargine } \\
\text { ic }\end{array}$ & - & $T 19$ & - & $\overline{5} 5$ & - & $\overline{0.9}$ & - & 70 & 52 & 35 & $\overline{0.6}$ & 53 & 48 & $\overline{-}$ \\
\hline . & Tyrosine & 0.04 & 1.3 & 0.01 & 0.2 & 0.04 & 0.9 & 0.10 & 0.9 & 2.5 & 0.8 & $\begin{array}{l}0.6 \\
0.1\end{array}$ & 2.1 & 2.4 & $\begin{array}{l}4.5 \\
1.5\end{array}$ \\
\hline Sulfur & Phenylalanine & 0.02 & 0.6 & 0.22 & 5.3 & - & - & 0.04 & 0.3 & 2.7 & 2.7 & 0.5 & 3.2 & 2.4 & 3.0 \\
\hline S Surrur & Cysteine & $0 \overline{09}$ & 2.6 & 0.08 & 2.0 & - & $\overline{-}$ & - & $\overline{-}$ & $\bar{z} \cdot+\cdot+r \cdot r$ & 0.3 & 0.3 & 2.6 & 0.8 & 0.5 \\
\hline & Methionine & & & & & - & & - & & $m$ & 0.3 & 0.3 & 2.6 & 0.8 & 0.5 \\
\hline Other & & & $\begin{array}{l}1,3 \\
0.7\end{array}$ & 001 & 0.5 & 0.04 & $\begin{array}{l}9.1 \\
0.8\end{array}$ & 011 & 5.7 & 1.4 & $\bar{z}$ & $=$ & $\bar{z}$ & $\bar{z}$ & $\bar{z}$ \\
\hline & Citrulline & 0.01 & 0.2 & - & 0.3 & -0.04 & - $-x$ & -1 & 0.1 & $=$ & $\bar{z}$ & 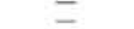 & $=$ & $=$ & $\bar{z}+\mathrm{r}+\mathrm{r}$ \\
\hline & Cystathionine & & - & - & - & - & - & - & - & $\overline{-}$ & - & - & - & - & $=$ \\
\hline & $\beta$-alanine & - & - & - & - & 0.10 & 2.1 & 0.74 & 4.6 & 0.9 & - & - & - & - & - \\
\hline & $\begin{array}{l}\alpha \text {-aminno butryic acid } \\
\beta \text {-amino iso-butyric acid }\end{array}$ & $=$ & 二 & z & $\bar{z}$ & 0.11 & 2.3 & $\overline{0.05}$ & $\overline{0.3}$ & $=$ & $=$ & $=$ & $=$ & $=$ & $\bar{z}+\mathrm{r}+\mathrm{r}$ \\
\hline & $\begin{array}{l}\gamma \text {-amino butyric acid } \\
\text { anposid }\end{array}$ & $\overline{011}$ & - & $\overline{0} \overline{01}$ & $\overline{0}$, & 0.19 & 4.0 & 0.01 & 0.1 & 0.5 & $\bar{z}$ & $=$ & - & $\bar{z}$ & $=$ \\
\hline Total & 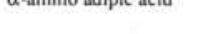 & 3.41 & $10 \overline{0}$ & 4.21 & $100^{2}$ & $\overline{4.78}$ & $10 \overline{0}$ & 1595 & $1 \overline{0}$ & $\overline{98.0}$ & 93.0 & 91.8 & 95.5 & 89.3 & 99.7 \\
\hline
\end{tabular}

Note: $-=$ not reported.

"Ittekkot et al. (1984a, 1984b)

"Peake et al. (1972).

${ }^{c}$ Degens $(1970$

${ }^{\mathrm{d}}$ King (1974). 